\title{
Effectiveness of manufactured surgical masks, respirators, and home-made masks in prevention of respiratory infection due to airborne microorganisms
}

\author{
Stephen Rossettie BA, Cody Perry BS, Mohammed Pourghaed BS, Mimi Zumwalt, MD
}

\begin{abstract}
According to a Centers for Disease Control (CDC) estimate, the health sector would require about 1.5 billion face masks plus 90 million respirators, and the public would need around 1.1 billion masks for a six-week influenza pandemic. ${ }^{1}$ As the current COVID-19 pandemic unfolds, concerns have been raised over depletion of medical supplies, including face masks. This has led to recommendations for healthcare personnel (HCP) to apply extended use and limited re-use strategies with face masks. ${ }^{2}$ In addition, the general population's response is mounting with persons making their own face masks for self-protection. This article aims to provide more detailed scientific information regarding the effectiveness and reusability of medical/surgical masks, respirators, and homemade masks.

Data have been collected from various journals and different studies listed in PubMed and the Cochrane Library. The CDC and the World Health Organization's (WHO) guidelines were also used extensively, as well as broader web searches of the English literature for up to date information.

Several investigators have shown that respirators are effective for reducing HCP exposure to airborne viruses and bacteria. ${ }^{3-6}$ Although some studies indicate no difference between the effectiveness of surgical masks and respirators, evidence does exist showing that respirators should be used instead of surgical masks when performing aerosol-generating procedures, since a respirator's tight seal and regulator are better equipped for minimizing airborne transmission. ${ }^{6,7}$ However, surgical masks are still considered an effective form of PPE since they provide some respiratory protection and can prevent the transmission of contagious respiratory droplets by infected individuals. As for the effectiveness of cloth homemade masks, this is difficult to determine since limited randomized controlled trials (RCTs) exist involving the use of these masks. ${ }^{8}$ However, studies conducted in some laboratory settings and one clinical investigation indicate that they provide minimal respiratory protection. ${ }^{9-11}$ It is important to continue further inquiries about the efficacy and reusability of surgical masks, respirators, and homemade masks, so that both healthcare providers and the public can collaborate to help reduce the destructive impact of SARS-CoV-2 and work toward improving the prevention of this highly infectious, potentially deadly transmissible disease.
\end{abstract}

Keywords: COVID-19, SARS-CoV-2, surgical masks, N95 respirator, cloth masks, effectiveness, reuse

Corresponding author: Mimi Zumwalt

Contact Information: Mimi.Zumwalt@ttuhsc.edu

DOI: $10.12746 /$ swrccc.v8i34.675 


\section{INTRODUCTION}

Paintings during the Renaissance era and of the city of Marseilles, France, the epicenter of the bubonic plague in 1720, depict mankind's first documented use of "medical masks." Gravediggers held handkerchiefs to their noses and doctors wore infamous long birdbeaked masks over their faces to protect themselves from the gases emanating from the ground known as miasma (Figure 1). Although now considered faulty logic, at the time it was widely believed these atmospheric stenches were the source of medical illnesses. Regardless, this idea stimulated innovative thoughts/ procedures that would eventually lead to today's manufactured face masks. ${ }^{12}$

By the late 1870s, this concept of miasma disappeared with the emergence of microbiology. The discovery of bacteria illuminated the spreading of illnesses and began the transition into today's modern medicine. By 1897, physicians began wearing the first "surgical masks", a plain handkerchief tied around the nose and mouth, to prevent germs from spreading through coughing and sneezing. Since then until modern day, this remains a critical concept that distinguishes a surgical mask from a respirator; a surgical mask is meant to protect the patient from germs, while a respirator is made for protecting the wearer. ${ }^{12}$

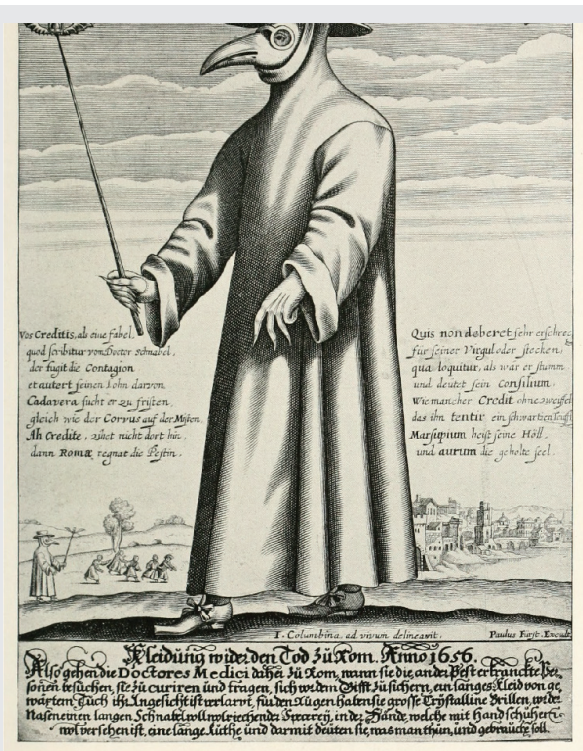

Figure 1. Dr. Schnabel, a 17th century plague doctor.
The first respirator was developed in 1910 due to an ongoing plague epidemic across Manchuria, a political complex jurisdiction shared between China and Russia. With a case fatality rate (CFR) near $100 \%$, both countries embarked in a scientific arms race to stop the spread of the plague; whichever nation showcased its intellectual capability first by protecting the people would win sovereignty over Manchuria. A medical school graduate of Chinese descent from Cambridge University, Dr. Lien-teh Wu expanded on the first looselyfitting surgical masks by designing a tighter fitting mask from gauze and cotton with multiple layers of cloth to better filter inhaled particles. Amid constant competition amongst his peers and after many empirical tests, his invention distinguished itself amongst the scientific community as an effective medical face mask. By early 1911, Dr. Wu's masks were worn everywhere by medical personnel, soldiers, and common citizens to fight against bacterial infections. It became the new international symbol of modern medicine by global expansion, subsequently seen following epidemics such as the Spanish Flu of 1918. Dr. Wu's template respirator design continued to evolve over time: air-filtering gas masks used during World War I and II, the first N95 singleuse "dust" respirator, and today's N95 electrostatically charged respirator utilized for protection during the current pandemic, COVID-19. ${ }^{12}$

Coronaviruses (CoV) are a large group of positivesense, single-stranded RNA viruses causing respiratory symptoms, possessing severity and virulence ranging from the common cold to serious pandemics such as Middle East Respiratory Syndrome (MERSCoV), Severe Acute Respiratory Syndrome (SARSCoV-1), and most recently COVID-19 (SARS-CoV-2) infection. To clarify, SARS-CoV-2 is the technical term for the virus that causes the infectious disease named COVID-19. Coronaviruses are zoonotic, meaning the main route of transmission is from animals to people. Each of the three coronavirus strains originated in bats then was transferred to an intermediate host before infecting humans. The intermediate hosts for each are thought to be civet cats, camels, and pangolins in SARS-CoV-1, MERS-CoV, and SARS-CoV-2, respectively. ${ }^{13}$

Despite its origin from wild animals, SARS-CoV-2 is highly contagious with human to human transmission 


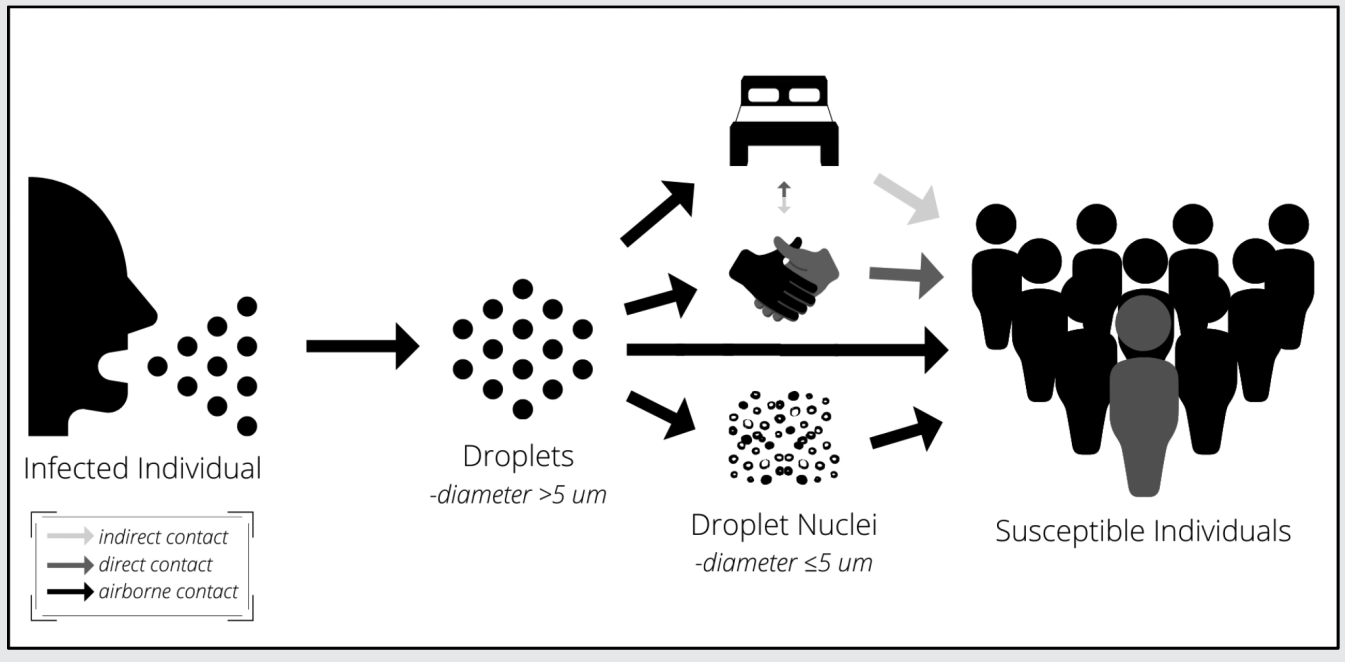

Figure 2. Transmission of Airborne Pathogens. occurring mainly via respiratory droplets and close direct contact (Figure 2). Although not confirmed by the CDC, the virus has also been shown in laboratory settings to be viable and transmissible in aerosolized form for as long as three hours and alive on solid surfaces for up to three days. ${ }^{14,15}$ In this experiment, the virus was more stable on plastic and stainless steel than on copper and cardboard. Limited evidence suggests the virus can also spread via fecal-oral transmission as well. ${ }^{16,17}$

As of March 27, 2020, the CDC estimates an average mortality rate of $\sim 2 \%$ worldwide from COVID-19, with 1,246 total deaths and 85,356 total cases in the United States. ${ }^{18}$ The R0 for the COVID-19 pandemic has been estimated to be between 2.2 and 3.58, meaning that on average, each person infected with the disease has spread it to 2-4 more people. ${ }^{19}$ In comparison, MERS-CoV totaled 857 official cases and 334 deaths, showing a high mortality rate of approximately $35 \%$ but much lower infectivity than COVID-19. On the other hand, COVID-19 appears to be more dangerous in both mortality and infectious rate than influenza, which has a mortality rate of about $0.1 \%$ and an $\mathrm{R} 0$ of $1.3 .{ }^{20}$ Additionally, risk is significantly increased with COVID19 in people with a BMI $\geq 40$, with immunosuppression, older than 65 years of age, or having comorbidities of the respiratory, renal, hepatic or cardiovascular system. In the elderly 85 years old and older, the mortality rate is estimated to be as high as $10-27 \%{ }^{21}$
The combination of these epidemiological factors poses a significant threat to our hospital systems. Furthermore, overcapacity and increased risk of transmission between caretakers and patients, many of whom present to the hospital with significant comorbidities, could make the virus even more lethal. Protocols for defense against the spread of this infectious disease include social separation (self-isolation), frequent adequate hand washing/sanitizing, and the use of personal protective equipment (PPE). However, isolation is not always possible for caretakers or patients in hospitals, thus the availability and proper use of PPE become substantially more important. ${ }^{22}$

\section{What is Personal Protective Equipment?}

Personal protective equipment (PPE) is the term for garments/devices worn to minimize exposure to hazards that cause serious workplace injuries and illnesses. ${ }^{23}$ As with any type of PPE, the key to proper selection and utilization of protective clothing, etc., is to understand both the hazards and risks of exposure. Important factors to consider when assessing hazardous exposure in healthcare facilities include the source, modes of transmission, physical pressures, types of contact, duration, and modes of tasks to be performed by the PPE user. The most commonly used forms of PPE are gloves, gowns/aprons, goggles, face shields, and masks or respirators. ${ }^{24}$ 
PPE has been shown to be very effective for deterring the spread of infection, but manufacturing standards necessitate more precision for protection against viral versus bacterial transmission. Due to the smaller size of viruses relative to bacteria, it is important that people use the correct types of PPEespecially masks and/or respirators - in order to prevent unnecessary transmission of small aerosolized particles. Thus, to reduce the spread of COVID-19, the CDC has recommended that healthcare personnel (HCP) treating suspected or confirmed COVID-19 patients wear non-sterile disposable patient examination gloves, surgical gowns, and a respirator that is at least as protective as a fit-tested N95 respirator. ${ }^{1,24}$

Considering the rapid spread of COVID-19 and subsequent shortages of PPE including respirators, the CDC has instructed HCP to use surgical masks instead of N95 respirators and to implement extended use and limited re-use of face masks when necessary. ${ }^{2}$ During this transition from conventional capacity strategies to contingency and critical capacity strategies, many questions arise as to the safety and efficacy of the various types of masks and respirators in protecting HCP from COVID-19. ${ }^{25}$

\section{Understanding SuRgical Masks AND RESPIRATORS}

In this section, key concepts will be discussed regarding the differences between surgical masks and respirators. In particular, comparison of the function, material, principles, and clinical effectiveness of each mask will be made. Subsequently, reuse of face masks will be discussed before the topic of homemade masks.

\section{FUNCTION}

A surgical mask is an infection control device designed to help prevent the spread of infection from the wearer's exhaled breath to potentially susceptible individuals. ${ }^{26}$ A surgical mask may help reduce contamination of the environment by providing a barrier for large droplets expelled by the wearer. ${ }^{27}$

In the context of COVID-19, surgical masks may be used for several different purposes, including being placed on suspected or confirmed COVID-19 patients to limit the spread of infectious respiratory secretions to others; they are worn by HCP to protect themselves from splashes or sprays of blood or bodily fluids. These face masks may also help the wearer to keep contaminated fingers/hands away from the mouth and nose. ${ }^{28}$

Despite these benefits, surgical masks are not designed to seal tightly against the user's face, which means that during inhalation, much of the potentially contaminated air can pass through gaps between the face and the mask edge, circumventing the effective functioning of the mask's filter material. As a result, surgical masks cannot be relied upon to completely protect patients or medical workers against airborne infectious agents. ${ }^{28}$

In contrast to surgical masks, respirators are made to filter out airborne particles, such as viruses and bacteria, by creating a tighter seal around the mouth and nose. (Refer to Figure 3 for more information about the differences between surgical masks and respirators). These devices should be worn in cases in which patients have confirmed viral infections, such as COVID-19, or when particulate matter, vapor, or gas are present. Respirators are manufactured with different grades including N95, N99, N100, depending on the percentage of $0.3 \mu \mathrm{m}$ particles to be filtered out (in the case of N95 respirators, at least 95\% of particles are stopped by the mask). An assigned protection factor (APF) may be designated on respirators according to the respirator's expected ability to reduce contamination when complying with an effective respiratory protection program. ${ }^{29}$ For example, an APF of 10 means a mask can be safely used in an atmosphere up to 10 -fold more contaminated than the Permissible Exposure Limit (PEL). N95 masks have been assigned an APF of $10 .^{30,31}$

\section{MATERIAL}

Surgical face masks are manufactured into a multilayered structure, generally by covering a layer of textile with non-woven bonded fabric on both sides. Nonwoven materials, which are inexpensive to make and clean due to their disposable nature, are made up of 
three or four layers. These disposable masks are often fabricated with two filter layers effective at filtering out extremely small particles such as bacteria above $1 \mu \mathrm{m}$ in size. It should be noted, however, that the filtration level of a mask depends on the type of fiber, the manner by which the fabric is manufactured, the web's structure, and the fiber's cross-sectional shape. For example, the material most commonly used to make surgical masks is polypropylene, either 20 or 25 grams per square meter (gsm) in density; however, masks can also be made from polystyrene, polycarbonate, polyethylene, or polyester composition. The $20 \mathrm{gsm}$ mask material is made from a spun-bond process, which involves extruding the melted plastic onto a conveyor. The material is extruded into a web, in which strands bond with each other as they cool. On the other hand, the $25 \mathrm{gsm}$ fabric is made via melt-blown technology, a similar process by which plastic is extruded through a die with hundreds of small nozzles and blown by hot air to become tiny fibers, again cooling and binding on a conveyor belt. After these manufacturing steps, masks are then stamped with nose strips, ear loops, and other attached pieces such as ties. ${ }^{30,32}$

Respirators, similar to surgical masks, consist of multiple layers. The outer layer on both sides is a protective non-woven fabric between 20 and $50 \mathrm{gsm}$ density to create a barrier both against the outside environment plus on the inside, to protect from the wearer's own exhalations. A pre-filtration layer then follows, which can be as dense as $250 \mathrm{gsm}$. This is usually a needled non-woven structure which is produced through hot calendaring, during which plastic fibers are thermally bonded by running them through high pressure heated rolls. This makes the pre-filtration layer thicker and stiffer to form while maintaining the desired shape. The innermost layer is a high efficiency melt-blown electric non-woven material, which determines the level of filtration efficiency. ${ }^{30}$

In order to capture particles, respirators rely on non-woven fibrous filter. Fibers from less than $1 \mu \mathrm{m}$ to $100 \mu \mathrm{m}$ in size crisscross to form a web of many layers, which is mostly air due to the spaces between the fibers. The aerated pockets between solid portions allow for breathability. Particulate matter is trapped, or captured, when flowing through the layers of filter media due to a number of different mechanisms including gravitational settling, inertial impaction, interception, diffusion, and electrostatic attraction. These physical principles provide the foundation for the efficacy of respirators in protecting HCP from infectious agents such as the SARS-CoV-2 virus. ${ }^{27}$

\section{Principles of face mask manufacturing AND SPECIFICATIONS}

Once assembly is completed, a quality assessment procedure is completed according to the American Society for Testing Materials (ASTM), an international certification organization for equipment manufacturing. Subsequently, masks must be approved by the Food and Drug Administration (FDA) for use during surgery. ${ }^{29}$ The masks are evaluated using five performance parameters: ${ }^{33,34}$

1. Bacterial filtration efficiency in vitro (BFE). Staphylococcus aureus aerosols, the leading nosocomial (hospital originating) infection, are shot through a mask using a particle size of $3.0 \mu \mathrm{m}$ and a flow rate of $28.3 \mathrm{~L} / \mathrm{min}$. The ratio of upstream staphylococcus aerosols to downstream residual concentration corresponds to the BFE with a high percentage indicating better performance.

2. Particle filtration efficiency (PFE). Polystyrene latex (PSL) microsphere aerosols are shot through a mask using a particle size of $0.1 \mu \mathrm{m}$ and a flow rate of $28 \mathrm{~L} / \mathrm{min}$. Similar to BFE calculations, the ratio of upstream PSL aerosols to downstream residual concentration equals the PFE with a high percentage indicating better performance.

3. Breathing resistance. Airflow is controlled, and the pressure difference is measured over the mask's surface area to determine its resistance to airflow. A low breathing resistance indicates better user breathability and mask shape maintenance.

4. Splash resistance. High velocity streams of fluid are shot through a mask using human blood pressures of 80,120 , and $160 \mathrm{mmHg}$ to simulate blood and other bodily fluid impact. The fastest of these three velocities that does not allow fluid penetration to the opposite side of the mask is then assigned a number. 
5. Flammability. Masks are set on fire and the time it takes for their flames to spread is measured. They are subsequently classified from class 1 to 4 depending on different organizations' criteria with class 1 indicating the least flammability.

Following the results of the mask's barrier properties, they are classified according to ASTM guidelines into 3 categories: low barrier (level 1), moderate barrier (level 2), and high barrier (level 3). ${ }^{33,34}$

- Level 1 masks have a BFE $\geq 95 \%$, no PFE requirement, and a fluid resistance of $80 \mathrm{mmHg}$; they also have a class 1 flammability, similar across all three levels. These masks are the main standard for surgical masks and are used during low-risk aerosol, fluid, or potential spray situations.

- Level 2 masks have a BFE $\geq 98 \%$, PFE $\geq 98 \%$, and a fluid resistance of $120 \mathrm{mmHg}$. They tend to be utilized for protection in light to moderate aerosol, fluid, or spray scenarios.

- Level 3 masks have a BFE and PFE $\geq 98 \%$, similar to Level 2, but a fluid resistance of $160 \mathrm{mmHg}$. They are designed for usage during moderate to heavy aerosol, fluid, or spray situations.

Minimum performance masks such as surgically molded utility masks fall below classification standards, while respirators are specified by different criteria than surgical masks, thus preventing direct comparison. Respirators fall under the National Institute for Occupational Safety and Health $(\mathrm{NIOSH})$ rather than ASTM and are tested using different procedures for PFE. In particular, respirators are first preconditioned for 24 hours at $85 \%$ relative humidity and $38^{\circ} \mathrm{C}$. They are then shot with sodium chloride, an aerosol charged-neutralized rather than un-neutralized, using a particle size of $0.3 \mu \mathrm{m}$ and a flow rate of $85 \mathrm{~L} /$ min. ${ }^{32}$ Thus, any claims directly comparing the "filtration efficiency" of a surgical mask to a respirator should therefore be ignored, since safety testing is not the same. ASTM-certified surgical masks exhibit a wide range of PFE, with the majority less than $70 \%$ when tested under NIOSH procedures. ${ }^{35}$ As a result, OSHA has not assigned protection factors to surgical masks and recommends $\mathrm{NIOSH}$ compliant respirators in circumstances dealing with airborne hazards. ${ }^{30}$

Sanchez has conducted notable work on the filtration efficiency of surgical masks using different particle sizes for evaluation under $\mathrm{NIOSH}$ guidelines. ${ }^{36}$ Double strap surgical masks with three layers of filter medium were tied onto a mannequin's face and either sealed on the edges with silicone or left unsealed. Similar to standard NIOSH procedures, the surgical masks were shot at by using three different PSL particle sizes: $0.5 \mu \mathrm{m}, 1 \mu \mathrm{m}$, and $2 \mu \mathrm{m}$. As expected, the sealed masks, which left no gaps for unfiltered gas exchange, demonstrated better filtration: $47.02 \%$ for $0.5 \mu \mathrm{m}$ particles $(23 \%$ increase over unsealed), $76.74 \%$ for $1.0 \mu \mathrm{m}$ particles ( $8 \%$ increase over unsealed), and $78.75 \%$ for $2.0 \mu \mathrm{m}$ particles (10\% increase over unsealed masks). The sealed surgical masks provided better protection during all testing trials and showed improvement over a wide range of variability seen in the unsealed mask during the $0.5 \mu \mathrm{m}$ trials, indicating the necessity of a tightlysealed mask for more effective protection against submicron particles. ${ }^{36}$

This characteristic of a tight seal, in addition to more rigorous $\mathrm{NIOSH}$ standards, allows respirators to be considered superior to surgical masks in preventing airborne disease transmission. Nonetheless, proper usage of respirators, surgical masks, and even homemade masks all make it more difficult to transfer a virus to the mucous membranes on the mouth and nose after touching a fomite (an object with the virus); all of these have at least some minor protective function against large respiratory droplets. ${ }^{34}$ It is also important to note that while respirators are used mainly to protect the wearer from outside airborne particles, many also function to decrease contamination from the wearer to the environment in the same way that surgical masks help in reducing contaminated particles. This 2-way protection depends largely on the type of respirator; those with valves reduce work of breathing by making exhaling easier, but simultaneously also make it simpler for respiratory droplets from the wearer's breath to escape the mask. ${ }^{37}$

The difference between respiratory droplets and aerosols (also known as droplet nuclei) is based on 
the size of the particle. Particles larger than $0.5 \mu \mathrm{m}$ in diameter are considered respiratory droplets that may fall onto a surface due to the forces of gravity. Aerosols, on the other hand, are classified as particles smaller than $0.5 \mu \mathrm{m}$, the behavior of which is closer to Brownian motion, whereby molecular interaction is a more important factor for motion than gravity, thus allowing these particles to remain in the air longer. ${ }^{38}$

Some studies have shown that the SARS-CoV-2 virus is $~ 60-140 \mathrm{~nm}$ in diameter with the highest concentration of aerosolized particles ranging from 0.25 $1.0 \mu \mathrm{m}$ in diameter. ${ }^{36,37}$ This has led to concern that the small size of SARS-CoV-2 may allow the virus to pass through the respirator filters, which are tested for larger $0.3 \mu \mathrm{m}$ particles. To address this concern, a study using six N95 respirators found that all were able to filter particles smaller than $0.1 \mu \mathrm{m}$ with $94 \%$ efficiency or better. While this finding demonstrates only respirator efficiency under laboratory conditions with inorganic or non-biological aerosols, other studies support the extrapolation of these data to biologicals by showing that filtration efficiency is based on particle size rather than the nature of a particle (e.g., bacteria, virus, nonbiological). ${ }^{41,42}$ Furthermore, when looking at filtration mechanisms, the evidence that high filter efficiency at $0.3 \mu \mathrm{m}$ size translates well to high filter efficiency at smaller sizes (as in the case of SARS-CoV-2) does make sense. For larger particles (greater than $0.6 \mu \mathrm{m}$ in diameter), filtering works through the process of interception and inertial impaction. Inertial impaction occurs when a particle is unable to follow a streamline of air around a fiber due to its inertia and instead collides into the fiber. Interception, on the other hand, is when the particle holds on to the air streamline, but that streamline will bring the particle close enough to come in contact with the fiber. These mechanisms are less significant in particles smaller than $0.1 \mu \mathrm{m}$, such as SARS-CoV-2, since diffusion and van der Waals forces become more powerful at this level. At this size, random movements of air molecules collide with these tiny particles and cause them to wander across streamlines until they come in contact with a fiber, where electrostatic attraction stops them from passing further through the filter. $^{27}$
The details and findings in this section indicate that viral transmission should be deterred more effectively with the use of tightly-fitted respirators rather than loosely-fitted surgical masks. However, the application of this theory to clinical settings has not resulted in an absolutely clear distinction. ${ }^{27}$

\section{EFFECTIVENESS}

Respirators are manufactured and regulated to prevent the wearer from exposure to airborne contaminants, largely due to the tight fit on the face and higher filtering power. ${ }^{34}$ This is important when considering whether to use a surgical mask or respirator because several pathogens including the SARS-CoV-2 virus are thought to be spread (at least partially) via the airborne route. ${ }^{14,43}$ Moreover, there is evidence that up to $90 \%$ of aerosol particles may penetrate surgical masks, while N95 respirators filter $95 \%$ to $99 \%$ of aerosol particulate matter. With these data, one would expect that the use of respirators by HCP when seeing patients with COVID-19 would lead to significantly decreased rates of transmission. ${ }^{35}$

Unfortunately, the recency of the COVID-19 pandemic limits the available data on the effectiveness of masks and respirators on SARS-CoV-2 virus transmission. However, data about these face masks with other respiratory illnesses can be interpreted to determine possible clinical efficacy. For example, Soerokromo et al. supported the concept that in some cases respirators can lead to better protective outcomes than surgical masks. This study measured contamination control in both laboratory and healthcare settings with three cohorts: surgical mask wearers, N95 respirator wearers, and controls. Results showed that surgical mask users exhibited a lower protection efficacy compared to N95 users for bacterial infection (surgical mask wearer colonization: $5.3 \%$; N95 wearer colonization: $2.9 \%)^{3}$

While this study focused on bacterial transmission, it is likely more predictive to look at studies focusing on influenza since this microorganism shares similar characteristics with the SARS-CoV-2 virus: both cause respiratory infection, are transmissible through droplets and aerosol, also with similarity in particle size. ${ }^{37}$ 
One such study analyzing two randomized control trials (RCTs) shows a statistically significant decrease in laboratory confirmed influenza $A$ and $B$ in groups with continuous or targeted use (while performing aerosol-generating procedures such as intubations or when seeing a patient with known respiratory illness), i.e., wearing N95 respirator relative to groups using medical masks. ${ }^{7}$ However, there was no statistically significant difference between the continuous respirator use group and the targeted respirator use group. This may support the idea that the benefit of respirators over surgical masks is due mainly to filtration of airborne particles in high-risk situations involving aerosols. This conclusion, in combination with the overall scarcity and higher cost of respirators (\$0.63 USD), supports the policy of wearing respirators only on a targeted basis. ${ }^{7,8}$

In contrast with the above study, other investigators have presented less conclusive results regarding the transmission rates of influenza and respiratory illnesses according to mask type..$^{4-6}$ A systematic review by Offeddu et al. provided evidence regarding protective effects of both surgical masks and respirators against clinical respiratory illness and influenzalike illnesses but demonstrated no significant difference between these groups for viral infections. ${ }^{6}$
Likewise, an RCT by Radonovich et al. and a systematic review by Smith et al. also found no significant difference between these two groups. ${ }^{35,36}$ However, the same systematic review did find evidence that compared to surgical masks, N95 respirators were associated with less filter penetration, less face seal leakage, and reduced total inward leakage under laboratory experimental conditions. ${ }^{4,5}$

The difference between laboratory/manufacturing findings and clinical results in these studies may be explained by human behavior and the shared function of the surgical masks with respirators. For example, improper donning (putting on) of respirators could negate the advantage of the otherwise tight seal and improper doffing (taking off) could lead to contamination and transmission of the infectious disease. Additionally, the potential exists for lower compliance among certain sets of people using respirators because the increased work of breathing may cause discomfort. Lastly, airborne transmission involves a smaller mechanism of particulate matter being transmitted as compared to respiratory droplets or direct contact for many of these viruses, so most of the protection from respirators comes from a barrier between large droplets due to sneezes, coughs, etc., and a barrier against indirect (fomite-based) contact with mucous membranes. ${ }^{16,44}$

\section{Surgical Mask}

for surgery
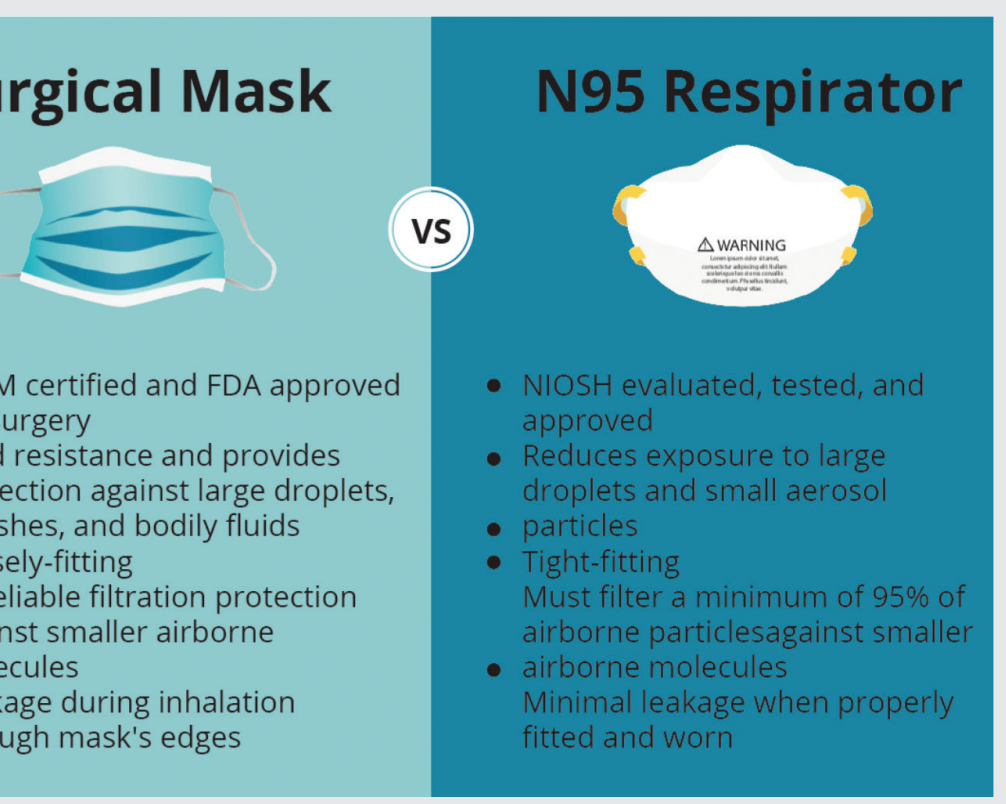

Figure 3. Adapted from Surgical Mask vs N95 Respirator (courtesy of CDC and NIOSH). 


\section{REUSE}

Optimizing strategies for the supply of face masks can vary depending on situation and demand. The CDC prioritizes three surge capacity approaches, dependent primarily on the quality of needed patient care and the availability of face masks: ${ }^{2}$

- Conventional Capacity. Standard daily practice for patient care and PPE administration.

- Contingency Capacity. Standard daily practice may be altered but quality of care remains insignificantly impacted. These practices can be used during expected periods of face mask shortage.

- Crisis Capacity. Practice and quality of care are significantly impacted and not representative of normal U.S. standards. These practices should be used during expected periods of known face mask shortage.

Normally, healthcare workers/others follow the conventional capacity strategy and go about their daily lives unaware of alternative approaches. Surgical masks are usually readily accessible to all individuals with no restrictions in hospital facilities. In the majority of settings, they are usually worn once and quickly disposed of in between uses. ${ }^{2}$

In the event of an abrupt, unanticipated increase in patient volume, healthcare systems may begin to experience strains in their facility space and face mask supply. This triggers the transition into a contingency capacity strategy in an attempt to prioritize materials while maintaining quality of care and supplies of face masks. Face masks then become restricted to HCP rather than left for public control in clinical settings, and people are urged to use disposable surgical masks and respirators for an extended period of time without removal. Reusable non-woven face masks made from traditional or micro-porous textiles can be another beneficial option since they can be sterilized and recycled up to 50 times, although it should be acknowledged that their barrier characteristics are inferior to disposable non-woven masks. ${ }^{32}$ In contrast, disposable masks cannot be cleaned since disinfectants eradicate their barrier properties and make the wearer more prone to infection. ${ }^{45}$
If patient volume continues to grow despite unresolved ongoing strain, as could occur with the COVID19 pandemic, the surge situation necessitates the switch to a crisis capacity strategy. At this point, HCP are advised to act conservatively with their masks and selectively choose when to wear them. Face masks then are recommended for use beyond their shelf lives and for multiple occasions due to supply shortages. Reusing face masks does increase the potential for contamination, but complete lack of protection may present greater infectious risks. Individuals reusing masks must consciously avoid touching their masks and carefully remove them in between uses to avoid contamination. Bacteria from the nasopharynx tract can accumulate on the filter and COVID-19 microparticles can potentially transmit onto the outer surface of a worn face mask. ${ }^{45}$ Furthermore, masks which are soiled, damaged, or difficult to breathe in should be immediately disposed of in all situations. ${ }^{2,30}$

\section{CLOTH AND HOMEMADE MASKS}

Cloth masks (see Figure 4), defined as "reusable masks made of cloth or any other fabric, including cotton, gauze, silk, or muslin," were first used in the late 19th century by patients to prevent the spread of infection. Updated and layered forms of the cloth masks were worn by HCP and the public throughout the 1900s during outbreaks of diphtheria, scarlet fever, and tuberculosis. The cloth masks were believed to lower rates of respiratory infections during these times. However, despite the use of cloth masks during the 1918 Spanish influenza pandemic, the number of influenza cases continued to rise. ${ }^{46}$ Cloth masks were studied moderately during the first half of the 20th century, but after the development of disposable surgical masks in the 1960s, very few studies have been conducted on the effectiveness of cloth masks. In addition, most of these studies have been conducted in a laboratory setting, although there is one clinical study performed in Vietnam that will be discussed in this section as well. ${ }^{8}$

According to an estimate by the CDC, approximately 1.5 billion masks plus 90 million respirators would be required by the health sector, and about 1.1 billion masks would be needed by the public for 


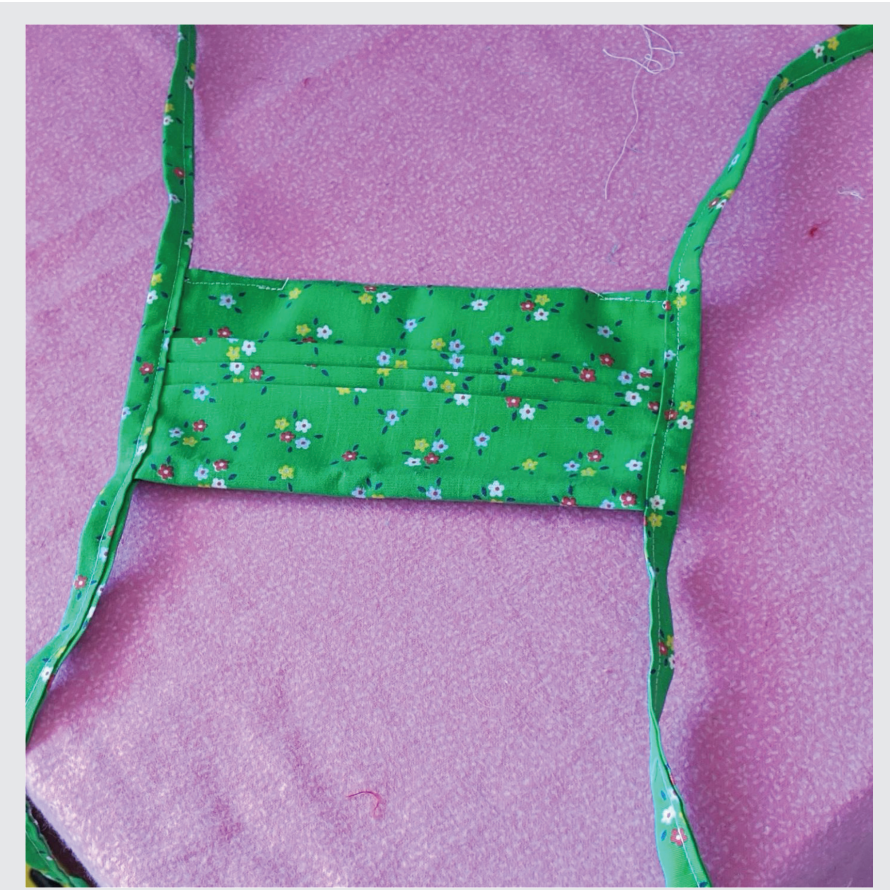

Figure 4. Homemade Cloth Mask (photo credit: Samantha Stanfield, MSc).

a six-week influenza pandemic. ${ }^{8}$ In light of the current rapidly depleting medical supplies nationally, concerns have been raised that HCP may run out of face masks. In such a shortage event the CDC has suggested the following options: ${ }^{2}$

1. Exclude HCP at higher risk for severe illness due to COVID-19 from contact with known or suspected COVID-19 patients.

2. Designate convalescent (clinically recovered) HCP for provision of care to known or suspected COVID19 patients.

3. Utilize a face shield that covers the entire front (extends to the chin or below) and sides of the face without a face mask.

4. Consider use of expedient patient isolation rooms for infectious risk reduction.

5. Consider usage of ventilated headboards.

6. Have HCP wear homemade masks.
Of interest is the CDC's provision that if no face masks are available, HCP might use homemade masks (e.g., bandana, scarf) as a last resort for the care of patients with COVID-19. However, the CDC also warns that homemade masks are not considered PPE, since their capability to protect HCP is unknown. In addition, the CDC recommends that masks made at home should ideally be used in conjunction with a face shield that covers the entire front and both sides of the face. Although the CDC offers homemade masks as an option, the World Health Organization (WHO) states that cloth (e.g., cotton or gauze) masks are not recommended under any circumstances. ${ }^{47}$ As a result of these conflicting guidelines from the CDC and the $\mathrm{WHO}$, it is necessary to provide more information about the efficacy of cloth masks. ${ }^{2}$

Although data are limited with regard to the use of cloth masks for HCP as PPE, some research has been conducted on the efficacy of cloth masks in protecting against pollution. Shayka et al. tested 3 different types of cloth masks for filtration efficiency against polluted particulates ranging in size from $30 \mathrm{~nm}$ to $2.5 \mu \mathrm{m}$, then compared these values to the filtration efficiency of surgical masks. These investigators found the filtration efficiency of cloth masks to be only $39-65 \%$ for polystyrene latex (PSL) microsphere aerosols, whereas the surgical masks performed statistically significantly better at 78-94\% efficiency against the same particles. This led researchers to conclude that disposable surgical masks are more effective than cloth masks in reducing particulate exposure, and that cloth masks are only marginally beneficial in protecting individuals from particles smaller than $2.5 \mu \mathrm{m} .{ }^{48}$ Due to the microscopic nature of bacteria and viruses, these results support both the WHO's caution against cloth masks and the CDC's guidelines for considering homemade masks as a "last resort".2,9

Three additional groups of researchers conducted mask testing in laboratory settings to further evaluate the effectiveness of cloth masks. A study by van der Sande et al. observed protective factors between respirators, surgical masks, and cloth masks during a short term inward, long term inward, and an outward protection experiment. They found that the FFP2 
(a form of respirator) and the surgical masks offered 50 times and 2 times as much protective effects during the inward protection experiments as homemade masks, respectively. These researchers also showed that FFP2 and surgical masks performed significantly better than the homemade masks in the outward protection experiment, although the FFP2 and surgical masks did not differ significantly from each other. These findings led these researchers to conclude that although all three products provide respiratory protection to a certain degree, homemade masks offer considerably less protection than respirators or surgical masks. ${ }^{9}$ Similar to van der Sande et al., Rengasamy et al. reported that cloth masks and other masks made from the fabric materials (cotton, polyester, cotton/polyester, polyester/nylon) commonly found in T-shirts, towels, and scarves offer minimal respiratory protection, since these homemade masks showed instantaneous penetration levels of $40-90 \% .{ }^{10}$

Following these studies, Davies et al. conducted experiments to determine the ideal material for homemade masks based upon filtration efficiency and pressure drop, then tested homemade masks in preventing the dispersal of droplets and aerosol particles produced by the wearer. In each of these tests, surgical masks were used as a control for comparison. To test the filtration efficiency of common household materials, a T-shirt (100\% cotton), scarf, tea towel, pillowcase, and vacuum cleaner bag were gathered for testing. Next circular cutouts of the tested materials were taken and placed without tension in airtight casings, creating a "filter" in which the material provided the only barrier to the transport of an aerosol. Next, a Henderson apparatus (a piece of equipment that allows for closed-circuit generation of microbial aerosols from a Collison nebulizer at a controlled relative humidity) was used to deliver the challenge aerosol across each material at $30 \mathrm{~L} / \mathrm{min}$. Downstream air was then sampled simultaneously for 1 minute into $10 \mathrm{ml}$ of phosphate buffer Manucol antifoam using 2 all-glass impingers. One impinger sampled the microorganisms that had penetrated through the material filter, while the other sampled the control (no filter). The collected fluid was removed from the impingers and assayed for microorganisms.
This test was performed 9 times for each material and the filtration efficiency of the fabric was measured. ${ }^{49}$

In addition to filtration efficiency, the pressure drop across the fabric was measured using a manometer (P200UL, Digitron), with sensors placed on either side of the filter casing, and was challenged with a clean aerosol at the same flow rate. ${ }^{49}$ The pressure drop across a mask is a useful measure of resistance to breathing. If respiratory protection is not capable of accommodating the breathing demands of the wearer, then it will impose an extra breathing load and cause discomfort. This is impractical especially for people with breathing difficulties. Furthermore, the extra breathing load may induce leakage due to the increased negative pressure in the face mask. ${ }^{50}$

To simulate particle challenge, Bacillus atrophaeus, a rod-shaped spore-forming bacterium (0.95$1.25 \mu \mathrm{m})$ and Bacteriophage MS2, a nonenveloped single-stranded RNA coliphage $(23 \mathrm{~nm})$ were used. This bacteria and virus were selected for two reasons. First, they are both known to survive the stresses caused by aerosolization. ${ }^{51,52}$ Second, the small size of the Bacteriophage MS2 and the relatively large size of $B$. atrophaeus provide an adequate range for comparison to the influenza virus, which is pleomorphic and ranges in size from $60-100 \mathrm{~nm} .{ }^{53}$ Each material was challenged with approximately $10^{7}$ colony-forming units (cfu) of $B$. atrophaeus and $10^{9}$ plaque-forming units (pfu) of bacteriophage MS2. ${ }^{49}$

Results from this study indicate that all the materials tested showed some capability to block the microbial aerosol challenges. In general, the masks' filtration efficiency for bacteriophage MS2 was $10 \%$ lower than $B$. atrophaeus. The surgical mask had the highest filtration efficiency $(90 \%)$ when challenged with bacteriophage MS2, followed by the vacuum cleaner bag $(86 \%)$. However, the bag's thickness created a high pressure drop across the material, rendering it unsuitable for a face mask. Similarly, the tea towel, a strong fabric with thick weave, showed a relatively high filtration efficiency $(83 \%$ with $B$. atrophaeus, $73 \%$ with bacteriophage MS2), but a high-pressure drop was also measured. The surgical mask measured a low 
pressure drop, and accordingly Davies et al. recommend it as the most suitable material for use as a face mask. That being said, the $100 \%$ cotton T-shirt had significantly lower filtration efficiencies against bacteriophage MS2 (50\%), but Davies et al. believe it to be the most suitable household material for an improvised mask for two reasons. First, unlike tea towels and vacuum cleaner bags, the T-shirt material demonstrated a low pressure drop. Second, T-shirts have a slightly stretchy quality to them, thus should provide a better fit to the user's face. ${ }^{49}$

As an additional note to this experiment, some who consider making or using homemade masks may predict that increasing the number of layers of fabric will improve results. Davies et al. tested this theory by doubling the layers of fabric in the T-shirt, tea towel, and pillowcase materials. Contrary to what one may expect, only the tea towel material demonstrated a significant increase in filtration efficiency, and all three fabrics exhibited an even greater pressure drop. These findings indicate that increasing the number of layers in homemade masks may not provide justifiable benefits, but more studies are needed to validate these findings and explore the possibility of using more $(3,4$, etc. $)$ layers. ${ }^{49}$

Since filtration efficiency is an indicator of inward mask penetration, it is also important to understand the effectiveness of homemade masks in preventing outward penetration and particle dissemination, which occurs during coughing and sneezing. To test outward penetration, Davies et al. recruited 21 subjects each of whom coughed with no mask on, while wearing a $100 \%$ cotton T-shirt based homemade mask, then a surgical mask. Outward penetration was measured in a mobile sampling chamber, or "cough box" using an Andersen sampler. Samples were then cultured on agar plates and total colony-forming units were counted. Results showed that both the surgical masks and homemade masks reduced the total number of microorganisms expelled when coughing, although the surgical mask was found to be generally more effective than the homemade mask. ${ }^{49}$

When taking into account the considerable differences in filtration efficiency and outward penetration,
Davies et al. emphasize that an improvised face mask should be viewed as the last possible alternative if the supply of commercial face masks is not available, irrespective of the disease against which it may be required for protection. At the conclusion of their article, these researchers issued a strong warning that homemade masks provide little protection from the microorganisms associated with respiratory diseases. ${ }^{49}$

Another concern about homemade face masks is how much the addition of a filter would improve the effectiveness of these masks. Because the studies regarding this question are limited, the topic requires discussion. Filters usually come in two forms: pleated hydrophobic (i.e., HEPA filter/HME, Sterivent Mini) or electrostatic (i.e., Clear-Therm, Filta-Therm). A study by Wilkes et al. revealed that pleated hydrophobic filters provide better bacterial and viral filtration performance against Bacillus subtilis var. niger aerosol under unconditioned (unused) and conditioned settings ( 24 hours of simulated use). Both unconditioned and conditioned masks had approximately a 90 times higher microbial penetration value (MPV) by viral challengers than bacterial challengers, although no significant difference existed between the unconditioned and conditioned settings themselves. ${ }^{54} \mathrm{~A}$ cost-tobenefit analysis must be performed as a recent survey indicated a majority of people did not believe the extra benefit of pleated hydrophobic filters outweighed its extra cost. ${ }^{49}$

Determining the effectiveness of face masks requires both an understanding of laboratory experiments and clinical outcomes. A cluster-randomized trial conducted by Maclntyre et al. allocated 74 wards from 14 hospitals (total of $1607 \mathrm{HCP}$ ) into three arms: medical masks at all times on their work shift; wearing cloth masks at all times on shift; or control (standard practice, which may or may not include mask usage). These wards were selected from high-risk settings (emergency, infectious/respiratory disease, intensive care units) for occupational exposure to respiratory infections, and outcomes were measured in terms of clinical respiratory illness (CRI), influenza-like illness (ILI), and laboratory-confirmed viral respiratory infection rates. The reported results showed that the 
infection rates for $\mathrm{CRI}, \mathrm{ILI}$, and laboratory-confirmed virus were $7.6 \%, 2.3 \%$, and $5.4 \%$ respectively for the cloth masks group, as opposed to infection rates of $4.8 \%, 0.2 \%$, and $3.3 \%$ respectively for the medical mask group. These findings indicated that infection rates were statistically significantly higher for respiratory illnesses in the cloth mask group in comparison to the medical mask group, which led Maclntyre et al. to caution against the use of cloth masks for HCP. However, this study was limited by a lack of measurement for compliance with hand hygiene, and the control subjects consisted of HCP wearing either medical masks or cloth masks, which may have introduced bias into the control group. In addition, the lack of other studies replicating similar findings makes it difficult to verify these results. Nonetheless, guidelines from the $\mathrm{WHO}$ and the $\mathrm{CDC}$, in vitro studies, and this clinical study provide reasons for the abundance of caution with regard to HCP and the use of cloth masks. ${ }^{11}$

Despite the lack of promise for cloth masks in laboratory or clinical experiments, some researchers believe that cloth masks still hold some merit. For example, cloth masks may help to prevent infected or symptomatic patients from spreading infective droplets into the environment. According to Scharman et al. and Bourouiba et al., even relatively large respiratory droplets can travel forward and upward by several meters. ${ }^{55,56}$ These droplets may become aerosolized, or end up depositing on and contaminating various surfaces, further spreading potentially infectious disease. ${ }^{57}$ Considering that the surface viability of coronaviruses can be several hours to a few days or longer, a cloth mask may help to reduce the spread of the SARS-CoV-2 virus by catching/containing the majority of respiratory droplets as they are expelled, which reduces fomites and the quantity of viral particles potentially being suspended in the air by a disease carrier. ${ }^{14,57,58}$

From a public health perspective, in addition to helping prevent the transmission of infectious disease, homemade cloth masks may also send messages encouraging positive behavior, e.g., promoting regular cleaning of face masks, avoiding the creation of an unwarranted sense of security, increasing likelihood for social distancing, discouraging face touching, and avoiding blockage in adoption of other, more effective means of protection. ${ }^{57,59}$

In summary, the WHO does not recommend the usage of cloth masks, while the CDC considers homemade masks as a last resort for HCP to use in critical times after complete exhaustion of other certified face masks. ${ }^{2,44}$ In vitro studies indicate that they offer poor filtration efficiency and minimal respiratory protection in comparison to respirators or surgical masks. ${ }^{9,10,49}$ From a clinical perspective, the study Maclntyre et al. conducted in Vietnamese hospitals provides fairly sound reason to believe that compared to surgical masks, cloth masks provide significantly less protection from respiratory illnesses. ${ }^{11}$ However, homemade masks may still hold a certain purpose for the lay public by reducing the transmission of infectious droplets and promoting positive health behaviors. ${ }^{14,58,59}$ Given that cloth masks are commonly used in low- and middle-income countries and are not adequately addressed within standardized infection control guidelines, more research is required to test the clinical efficacy of cloth masks. In addition, future research questions should include filtration efficacy (with and without a filter), length of use, methods of decontamination, and face fit testing. ${ }^{8}$

\section{LIMITATIONS}

The primary limitation of this article is an overall lack of data on SARS-CoV-2 specific transmission, due largely to the recent discovery of this novel virus. This results in an increased reliance on and extrapolation from studies that examined other viruses, such as influenza to determine mask effectiveness. Cloth masks have a similar paucity of data and a lack of replicated RCTs. Finally, limitations are also inherent in much of the literature cited, especially due to bias caused by concurrent interventions such as PPE and hand hygiene compliance. In many of these research studies, community acquired infection was not accounted for, so this could skew the data that are supposed to otherwise represent nosocomial infections. 


\section{ConCLUSION}

Although evidence/data are still being gathered on the effectiveness of face masks and other PPE for COVID-19, the major health and safety organizations consistently warn that people should be careful not to confuse the potential benefits of wearing a mask with the definitive and significant benefits of social separation/physical distancing. It is much safer to avoid the company of people who are sick or potentially infectious than it is to be in close contact with these people, regardless of the PPE worn.

Nevertheless, many essential workers in healthcare and other fields will have to continue with unwanted/ unintended exposure to people with the virus. Thus, it is important that they are well informed about the best possible practices in order to protect their own health and the health of those whom they care for. Several investigations have shown that respirators are effective for reducing HCP exposure to airborne viruses and bacteria. ${ }^{3-6}$ Although some studies indicate no difference between the effectiveness of surgical masks and respirators, other data demonstrate that respirators should be used over surgical masks when performing aerosol-generating procedures, as the tight fit and regulated filters in respirators are better equipped for deterring airborne transmission. ${ }^{6,7}$

However, surgical masks are still considered an important form of PPE, since they do provide some respiratory protection and can be placed upon infected individuals to prevent the transmission of contagious respiratory droplets. On the other hand, the effectiveness of cloth and homemade masks is difficult to determine since there are limited RCTs involving the use of these masks. ${ }^{8}$ However, studies conducted in some laboratory settings and one clinical investigation indicate that they provide minimal respiratory protection in comparison to surgical masks or respirators. It is important to study/continue further inquiries on the efficacy and reusability of surgical masks, respirators, and homemade face masks, so that both healthcare providers and the public can collaborate to help reduce the destructive impact of SARS-CoV-2 and work toward improving prevention of this highly infectious, potentially deadly transmissible disease..$^{9-11}$
Article citation: Rossettie S, Perry C, Mohammed Pourghaed M, Mimi Zumwalt M. Effectiveness of manufactured surgical masks, respirators, and homemade masks in prevention of respiratory infection due to airborne microorganisms. The Southwest Respiratory and Critical Care Chronicles 2020;8(34):11-26

From: The Department of Orthopedics (MZ) and the School of Medicine (SR, CP, MP), Texas Tech University Health Sciences Center, Lubbock, Texas

Submitted: 4/1/2020

Accepted: 4/5/2020

Reviewer: Kenneth Nugent MD

Conflicts of interest: none

This work is licensed under a Creative Commons Attribution-ShareAlike 4.0 International License.

\section{REFERENCES}

1. Reusability of facemasks during an influenza pandemic: facing the flu at NAP.edu. National Academies Press: OpenBook. The National Academies of Science Engineering Medicine; Accessed 3/26/2020. Available from: https://www.nap.edu/ $\mathrm{read} / 11637 / \mathrm{chapter} / 5$

2. Strategies for optimizing the supply of facemasks: COVID19. Centers for Disease Control and Prevention. Centers for Disease Control and Prevention; 2020. Accessed 3/25/2020. Available from: https://www.cdc.gov/coronavirus/2019-ncov/ hcp/ppe-strategy/face-masks.html

3. Soerokromo N, Retera L, Lankhorst N. Efficacy of face masks and respirators in preventing upper respiratory tract bacterial colonization and co-infection in hospital healthcare workers - Comment on the article by MacIntyrev. Preventive Medicine 2014;65:153.

4. Radonovich LJ, Simberkoff MS, Bessesen MT, et al. N95 respirators vs medical masks for preventing influenza among health care personnel: a randomized clinical trial. JAMA 2019 Sep 3;322(9):824-33. https://jamanetwork.com/journals/ jama/fullarticle/2749214

5. Smith JD, MacDougall CC, Johnstone J, et al. Effectiveness of N95 respirators versus surgical masks in protecting health care workers from acute respiratory infection: a systematic review and meta-analysis. CMAJ 2016 May 17;188(8):56774. https://www.semanticscholar.org/paper/Effectiveness-ofN95-respirators-versus-surgical-in-Smith-Macdougall/ 7cfdda149f4cde31c1c1fdd2ff34a62e4ef8703b

6. Offeddu V, Yung CF, Low MS, et al. Effectiveness of masks and respirators against respiratory infections in healthcare 
workers: a systematic review and meta-analysis. Clinical Infectious Diseases 2017 Nov 13;65(11):1934-42. https:// academic.oup.com/cid/article/65/11/1934/4068747

7. MacIntyre CR, Chughtai AA, Rahman B, et al. The efficacy of medical masks and respirators against respiratory infection in healthcare workers. Influenza and other respiratory viruses 2017;11(6):511-7. https://onlinelibrary.wiley.com/doi/full/10. 1111/irv.12474

8. Chughtai AA, Seale H, MacIntyre CR. Use of cloth masks in the practice of infection control-evidence and policy gaps. Int J Infect Control 2013;9(3).

9. Van der Sande M, Teunis P, Sabel R. Professional and homemade face masks reduce exposure to respiratory infections among the general population. PLoS One 2008;3(7).

10. Rengasamy S, Eimer B, Shaffer RE. Simple respiratory protection-evaluation of the filtration performance of cloth masks and common fabric materials against 20-1000 nm size particles. Annals of occupational hygiene 2010;54(7):789-98.

11. MacIntyre CR, Seale H, Dung TC, et al. A cluster randomised trial of cloth masks compared with medical masks in healthcare workers. BMJ open 2015;5(4):e006577.

12. Wilson M. The untold origin story of the N95 mask. Fast Company. Fast Company; 2020. Accessed 3/24/2020. Available from: https://www.fastcompany.com/90479846/the-untoldorigin-story-of-the-n95-mask

13. Zhang $\mathrm{T}, \mathrm{Wu} \mathrm{Q}$, Zhang Z. Probable pangolin origin of SARS-CoV-2 associated with the COVID-19 outbreak. Current Biology 2020 Mar 19.

14. Van Doremalen N, Bushmaker T, Morris DH, et al. Aerosol and surface stability of SARS-CoV-2 as compared with SARSCoV-1. New England Journal of Medicine 2020 Mar 17.

15. Chinese Center for Disease Control and Prevention. General questions: COVID-19 prevention and control.

16. Ong SW, Tan YK, Chia PY, et al. Air, surface environmental, and personal protective equipment contamination by severe acute respiratory syndrome coronavirus 2 (SARS-CoV-2) from a symptomatic patient. JAMA 2020 Mar 4.

17. Official Guidance. COVID-19: Guidance for infection prevention and control in healthcare settings. Version 1.0.: Department of Health and Social Care (DHSC), Public Health Wales (PHW), Public Health Agency (PHA) Northern Ireland, Health Protection Scotland (HPS) and Public Health England 2020. Accessed 3/26/2020. https://assets.publishing.service. gov.uk/government/uploads/system/uploads/attachment_ data/file/874316

18. Cases in U.S. Centers for Disease Control and Prevention. Centers for Disease Control and Prevention; 2020. Accessed 3/28/2020. Available from: https:/www.cdc.gov/coronavirus/ 2019-ncov/cases-updates/cases-in-us.html

19. He F, Deng Y, Li W. Coronavirus disease 2019 (COVID 19): what we know? Journal of Medical Virology 2020 Mar 14.
20. Sheikh $\mathrm{K}$, Watkins $\mathrm{D}, \mathrm{Wu} \mathrm{J}$, et al. How bad will the coronavirus outbreak get? Here are 6 key factors. The New York Times. The New York Times; 2020. Accessed 3/28/2020. Available from: https://www.nytimes.com/interactive/2020/ world/asia/china-coronavirus-contain.html\#virulence

21. People who are at higher risk for severe illness. Centers for Disease Control and Prevention. Centers for Disease Control and Prevention; 2020. Accessed 3/28/2020. Available from: https://www.cdc.gov/coronavirus/2019-ncov/specificgroups/people-at-higher-risk.html

22. What to do if you are sick. Centers for Disease Control and Prevention. Centers for Disease Control and Prevention; 2020. Accessed 3/26/2020. Available from: https:/www.cdc.gov/ coronavirus/2019-ncov/if-you-are-sick/steps-when-sick.html

23. United States Department of Labor. Safety and Health Topics | Personal Protective Equipment | Occupational Safety and Health Administration. Accessed 3/26/2020. Available from: https://www.osha.gov/SLTC/personalprotectiveequipment/

24. Frequently asked questions about personal protective equipment. Centers for Disease Control and Prevention. Centers for Disease Control and Prevention; 2020. Accessed 3/25/2020. Available from: https://www.cdc.gov/coronavirus/2019-ncov/ hcp/respirator-use-faq.html

25. Hick J, Barbera J, Kelen, G. Refining surge capacity: conventional, contingency, and crisis capacity. Disaster Medicine and Public Health Preparedness, 3(S1), S59-S67. doi: 10.1097/DMP.0b013e31819f1ae2

26. American Industrial Hygiene Association: Biosafety Reference Manual, 2nd ed., P.A. Heinsohn, R.R. Jacobs, and B.A. Concoby (eds.), Fairfax, VA: American Industrial Hygiene Association, 1996.

27. Respiratory protection for airborne exposures to biohazards. 3M. 2020. Available from: https://multimedia.3m.com/mws/ media/409903O/respiratory-protection-against-biohazards. pdf

28. United States Department of Labor. OSHA fact sheet: respiratory infection control: respirators versus surgical masks | Occupational Safety and Health Administration. Accessed 3/26/2020. Available from: https://www.osha.gov/ Publications/respirators-vs-surgicalmasks-factsheet.html

29. Assigned protection factors for the revised respiratory protection standard. Accessed 3/26/2020. Available from: https://www.osha.gov/Publications/3352-APF-respirators. html

30. Henneberry B. How surgical masks are made, tested and used. How Surgical Masks Are Made. Thomas; Accessed3/26/2020. Available from: https:/www.thomasnet.com/articles/other/ how-surgical-masks-are-made/

31. Center for Devices and Radiological Health. N95 respirators and surgical masks (face masks) [Internet]. U.S. Food and Drug Administration. FDA; Accessed 3/26/2020. Available from: 
https://www.fda.gov/medical-devices/personal-protectiveequipment-infection-control/n95-respirators-and-surgicalmasks-face-masks

32. Respirators and surgical masks: a comparison. 3M. 2020. Available from: http://multimedia.3m.com/mws/media/ 9577300/respirators-and-surgical-masks-contrast-technical-bulletin.pdf

33. Chellamani KP, Veerasubramanian D, Vignesh Balaji RS. Surgical face masks: manufacturing methods and classification. Journal of Academia and Industrial Research 2013; 2(6):320-4.

34. ASTM F2100-19: Standard specification for performance of materials used in medical face masks. West Conshohocken, PA: ASTM International; 2019. doi:10.1520/F2100-19.

35. Oberg T, Brosseau LM. Surgical mask filter and fit performance. American Journal of Infection Control 2008; 36(4):276-82.

36. Sanchez E. Graduate theses and dissertations. Graduate Theses and Dissertations. 2010. Available from: https:// scholarcommons.usf.edu/etd/1760

37. Campbell J. N95 vs FFP3 \& FFP2 masks - what's the difference? Fast Life Hacks. 2020. Accessed 3/26/2020. Available from: https://fastlifehacks.com/n95-vs-ffp/\#Valve_vs_ Non-Valved_Respirators

38. Wei NKWK, National University of Singapore, Smart Air. What is PM0.3 and why is it important? [Internet]. Smart Air Filters. 2020 Accessed 3/26/2020. Available from: https:// smartairfilters.com/en/blog/what-is-pm0-3-why-important/

39. Liu Y, Ning Z, Chen $Y$, et al. Aerodynamic characteristics and RNA concentration of SARS-CoV-2 aerosol in Wuhan hospitals during COVID-19 outbreak. bioRxiv 2020 Jan 1.

40. Zhu N, Zhang D, Wang $\mathrm{W}$, et al. A novel coronavirus from patients with pneumonia in China, 2019. New England Journal of Medicine. 2020 Jan 24. https://www.nejm.org/doi/ full/10.1056/NEJMoa2001017

41. Garner PD, Eshbaugh JP, Harpest SD, et al. Viable viral efficiency of N95 and P100 respirator filters at constant and cyclic flow. J Occ. Environ Hyg. 10(10):564-572;2013.

42. Harnish DA, Heimbuch BK, Husband M, et al. Challenge of N95 filtering facepiece respirators with viable H1N1 influenza aerosols. Infect. Cont. Hosp. Ep 34(5):494-499;2013.

43. Shine KI, Rogers B, Goldfrank LR. Novel H1N1 influenza and respiratory protection for health care workers. N Engl J Med 2009;361(19):1823-182519797278

44. Frequently asked questions. Centers for Disease Control and Prevention. Centers for Disease Control and Prevention; 2020. Accessed 3/26/2020. Available from: https://www.cdc. gov/coronavirus/2019-ncov/faq.html

45. Use and reuse of respiratory protective devices for influenza control. in: reusability of facemasks during an influenza pandemic: facing the flu. Washington, DC: The National
Academies Press; 2006. p. 42-56. Available from: https:// doi.org/10.17226/11637

46. Brooks WA. The open air treatment of influenza. The American Journal of Public Health 1918; 8: 276-750. http://dx.doi. org/10.2105/AJPH.8.10.746

47. World Health Organization. Advice on the use of masks in the community, during home care, and in health care settings in the context of COVID-19: interim guidance, 19 March 2020. World Health Organization; 2020.

48. Shakya KM, Noyes A, Kallin R, et al. Evaluating the efficacy of cloth facemasks in reducing particulate matter exposure. J Expo Sci Environ Epidemiol 2017;27(3):352-357. doi:10.1038/jes.2016.42

49. Davies A, Thompson K-A, Giri K, et al. Testing the efficacy of homemade masks: would they protect in an influenza pandemic? Disaster Medicine and Public Health Preparedness 2013 May 22;7(4):413-8.

50. Clayton MP, Bancroft B, Rajan B. A review of assigned protection factors of various types and classes of respiratory protective equipment with reference to their measured breathing resistances. Ann Occup Hyg 2002;46:537-547.

51. Cox, C. The aerobiological pathway of microorganisms. Chichester, UK: John Wiley \& Sons; 1987. Google Scholar

52. Dubovi EJ, Akers TA. Airborne stability of tailless bacterial viruses S-13 and MS-2. Appl Microbiol 1970;19:624-628. Google Scholar | PubMed

53. Stanley, WM. The size of influenza virus. J Exp Med 1944; 79:267-283.CrossRef | Google Scholar | PubMed

54. Wilkes AR, Benbough JE, Speight SE, et al. The bacterial and viral filtration performance of breathing system filters. Anaesthesia 2000;55(5):458-65.

55. Scharfman BE, Techet AH, Bush JWM, et al. 2016. Visualization of sneeze ejecta: steps of fluid fragmentation leading to respiratory droplets. Experiments in Fluids https://doi. org/10.1007/s00348-015-2078-4.

56. Bourouiba L, Dehandschoewercker E, Bush JWM. 2014. Violent expiratory events: on coughing and sneezing. Journal of Fluid Mechanics https://doi.org/10.1017/jfm.2014.88.

57. Svara F. Promoting simple do-it-yourself masks: an urgent intervention for COVID-19 mitigation. Medium; 2020. Accessed 3/25/2020. Available from: https://medium. com/@matthiassamwald/promoting-simple-do-it-yourselfmasks-an-urgent-intervention-for-covid-19-mitigation14da4100f429

58. Kampf G, Todt D, Pfaender S, et al. 2020. Persistence of coronaviruses on inanimate surfaces and their inactivation with biocidal agents. J Hospital Infection 104(3):246-51.

59. Wada K, Oka-Ezoe K, Smith DR. 2012. Wearing face masks in public during the influenza season may reflect other positive hygiene practices in Japan. BMC Public Health 12 (December): 1065. 